\title{
WHERE SHOULD WE MANAGE DIABETIC KETOACIDOSIS (DKA) IN CHILDREN?
}

\author{
S. Broughton, F. Williams, M. Ford-Adams
}

Child Health, Kings College Hospital NHS Foundation Trust, London, UK

Background: The leading cause of mortality for children with diabetes is DKA, with cerebral oedema the main cause of death. The onset of cerebral oedema is unpredictable; therefore the key to improving survival is early recognition and aggressive management.The British Society for Paediatric Endocrinology and Diabetes (BSPED)guidelines for DKA were updated in 2009; listing the recommended inpatient observations and emphasising "safe nursing" on general paediatric wards, acknowledging that children with DKA are high dependency patients and require 1:1 nursing.

Methods: We audited Paediatric DKA admissions in 2009 ( $\mathrm{n}=26,8$ admitted to HDU) comparing the quality of physiological data collection and outcome between those admitted to the general wards (1:4 nursing) and to $\mathrm{HDU}$ (1:2 nursing).

Results: The HDU patients were more acidotic ( $\mathrm{pH} 6.9$ (range 6.8-7.2) vs 7.2 (7.1-7.3)) $(\mathrm{p}=0.03$ ) and tended to be more likely to be new diagnoses ( $71 \%$ vs $41 \%$ ). Children with DKA on HDU had better recording of fluid balance, hourly blood sugars, physiological and neurological observations than those nursed on general paediatric wards.

Conclusions: Recording of physiological parameters in a HDU setting was more complete than in a general Paediatric setting. The BSPED guidelines recommend one to one nursing for children with DKA on Intravenous insulin, which may not be feasible in all units. Accurate observations and swift intervention are necessary to improve survival from cerebral oedema. Therefore children with DKA should be managed in a regional HDU or a large DGH with at least 1:2 nursing. 